\title{
Factor VII deficiency- A rare Case Report in Qatar
}

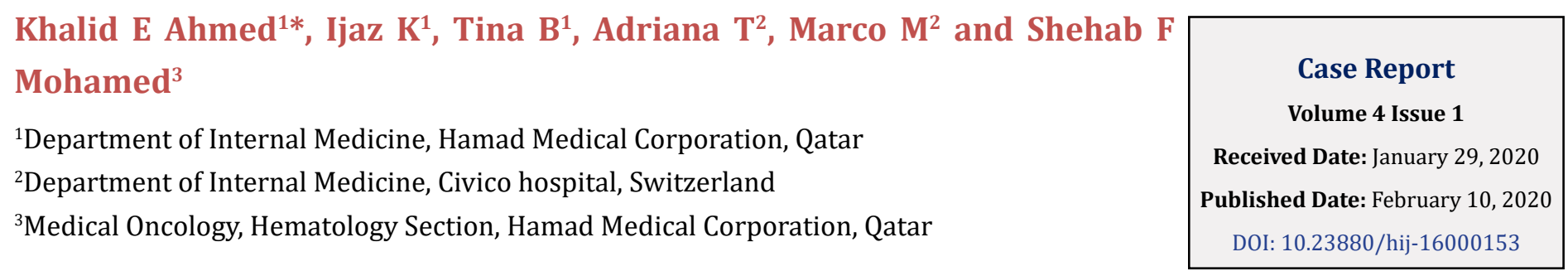

*Corresponding author: Khalid E Ahmed, Department of Internal Medicine, Hamad Medical

Corporation, Qatar-Doha-al Rayyan Street, Post box: 3050, Qatar, Tel: 0097466764033; Email: kahmed21@hamad.qa

\section{Abstract}

Factor VII deficiency is a rare inherited coagulation disorder. Elevated prothrombin time and INR with a normal partial thromboplastin time and bleeding time points towards an isolated Factor VII deficiency. We hereby report a case of a 22-years old Indian male who presented with bleeding gums following dental scaling. Labs supported the deficiency of Factor VII at less than 5.6\%. Infusion of recombinant Factor VIIa helped to normalize the prothrombin time and INR.

Keywords: Factor VII deficiency; Rare Inherited Coagulation Disorder; Bleeding Gums

Abbreviations: RICDs: Rare Inherited Coagulation Disorders; PT: Prothrombin Time; INR: International Normalized Ratio; aPTT: Activated Partial Thromboplastin Time; BT: Bleeding Time; PC: Platelet Count.

\section{Introduction}

Factor VII is a coagulation factor of the extrinsic pathway, which is involved in secondary hemostasis [1]. Deficiency of factor VII alone is very rare with a prevalence of 1 in 500,000 individuals, thus it falls under the category of rare inherited coagulation disorders (RICDs) [2]. It is the most common among the rare inherited coagulation disorders which also include factors XIII, XI, X, V and II.

We hereby present the case of a 22 years old Indian male with a newly diagnosed Factor VII deficiency.

\section{Case Presentation}

A 22 years old Indian male presented to the Emergency Department with bleeding gums of one day duration. The patient had dental scaling performed two days prior to the admission. The patient has not had similar episodes in the past. He reported an injury to his right shin a month prior to this presentation, which healed without any complications. He denied any chemical exposure or use of any medications or herbal supplements. He denied any previous hx of nose bleeding, joint bleeding, skin rash or spots. His family history is remarkable for his sister having bleeding problems, of which he does not know the details. He is single, non-smoker and does not consume alcohol. His physical examination was unremarkable except for obvious gum bleeding.

The investigations revealed a prolonged Prothrombin time (PT) at 96 seconds (Normal: 7.8-9.9seconds) and International normalized ratio (INR) of 9.4. Platelet count, Activated Partial Thromboplastin Time (aPTT), D-dimer and fibrinogen, were within normal limits. Further investigation revealed Factor VII deficiency with levels less than 5.6\% (normal is between 50\%- 150\%). All the other factors, including II, VIII, IX, X, XIII and vWF were within normal range. Thus, the patient was diagnosed with factor VII deficiency.

Initially the patient was treated with Fresh Frozen plasma, Vitamin $\mathrm{K}$ and tranexamic acid. When there was no 


\section{Haematology International Journal}

response to this treatment, he was given 4 doses of Factor VII $550 \mathrm{mcg}$. The patient responded to Factor VII and the bleeding stopped. Repeat hematologic studies revealed normal levels of PT and INR following treatment. The patient was discharged after appropriate education and counselling. As his parents do not live here therefore, he was advised to get his parents checked for the deficiency. He was given factor VII deficiency card for future surgeries and procedures.

\section{Discussion}

Factor VII deficiency is a rare disorder with incidence rate of $1 / 500,000$ people but the rate varies across regions. More cases are seen in regions with consanguineous marriages, given that it is inherited in autosomal recessive pattern. Turkey has higher than average rates, accounts for around one third of the cases with rare inherited clotting disorders [3].

Factor VII is a vitamin $\mathrm{K}$ dependent factor synthesized in the liver. Factor VII has extremely short half-life compared to other coagulation factors- 3-4 hours only. Five allelic polymorphisms have been identified in factor VII gene, which can affect the activity of the factor by almost 25-30\% [4]. Other factors affecting the activity of factor VII include environmental factors like dietary fat, age, obesity and sex hormones.

Clinical manifestation of factor VII deficiency varies among different individuals. It doesn't have any correlation with the mutations or the amount of Factor VII in circulation. In a study performed across Europe and Latin America, 71\% of individuals with homozygous mutation in Factor VII were asymptomatic while $50 \%$ of individuals with compound heterozygosity were symptomatic [5].

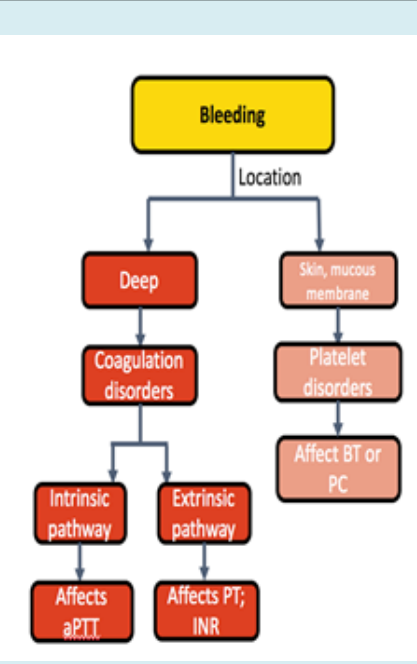

Figure 1: Initial approach to patient with bleeding disorder.
Deficiency of Factor VII can manifest with skin and mucosal bleeding- as seen in our patient, e.g. epistaxis, menorrhagia, etc. which are more commonly associated with defects in primary hemostasis (Figure 1). Factor VII deficiency can also manifest in infancy, mostly as GI and CNS bleeding. CNS bleeds usually present in infants with severe factor VII deficiency commonly as intracerebral hemorrhages and they have high morbidity and mortality [6]. In patients with levels less than $20 \%$, spontaneous bleeding is seen. Our patient had less than $5.6 \%$ of Factor VII, thus presenting as a case of spontaneous bleeding.

Bleeding complications related to surgical interventions are usually low with Factor VII deficiency. In cases where the deficiency is severe, a mere increase in the plasma concentration of Factor VII to $10-15 \mu / \mathrm{dL}$ is enough to prevent any bleeding complications. The age at the time of manifestation of clinical symptoms also varies across individuals. The median age at diagnosis is seven years with a range from 0 (infancy) to 73 years old [7]. It affects both genders equally.

Treatment options for patients with rare inherited bleeding disorders depend on the symptoms. If the patients are asymptomatic, no treatment is required. Current therapeutic options for symptomatic Factor VII deficiency include recombinant factor VII (rFVIIa), prothrombin complex concentrate (PCC), fresh frozen plasma, and fibrinolytic inhibitors.

Among these, the treatment of choice is recombinant factor VIIa. With a dose of 20-25 $\mu \mathrm{g} / \mathrm{kg}$ administered every 4-6 hour for any bleeding complications or surgical interventions in patients with Factor VII deficiency. The major limitation in providing this treatment is the availability of rFVIIa. Prophylactic treatment is generally not recommended in patients with Factor VII deficiency.

\section{Conclusion}

Congenital factor VII deficiency is a rare congenital disorder which should be suspected in any individual with atypical clinical presentation of bleeding symptoms and elevated PT and INR with normal levels of aPTT.

Conflict of Interest: The authors declare that they have no conflict of interest with regard to the publication of this article.

\section{References}

1. Mir MA (2019) Factor VII Deficiency.

2. Peyvandi F, Menegatti M (2015) Rare Inherited Coagulation Disorders. Postgraduate Haematology, pp: 
733-742.

3. Moerloose P, Schved JF, Nugent D (2016) Rare coagulation disorders: fibrinogen, factor VII and factor XIII. Haemophilia 5: 61-65.

4. Perry DJ (2002) Factor VII Deficiency. British Journal of Haematology 118(3): 689-700.

5. Hartung HD (2017) Pediatric Factor VII Deficiency.
6. Herrmann FH, Wulff K, Auerswald G, Schulman S, Astermark J, et al. (2009) Factor VII deficiency: clinical manifestation of 717 subjects from Europe and Latin America with mutations in the factor 7 gene. Haemophilia 15(1): 267-280.

7. Peyvandi F, Palla R, Menegatti M, Mannucci PM (2016) Introduction: Rare bleeding disorders: general aspects of clinical features, diagnosis, and management. Semin Thromb Hemost 35(4): 349-355.

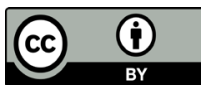

